\title{
Surface Marking a Helpful Technique in the Approach of Acromioclavicular Joint During Shoulder Arthroscopy
}

\section{Munawar Shah ${ }^{1 *}$, Usama Bin Saeed ${ }^{2}$, Mohammed Sufyan ${ }^{3}$, Danial Shah and Kishen Parekh ${ }^{4}$}

${ }^{1}$ Consultant Orthopaedic Surgeon, Walsall Manor Hospital, West Midlands, UK

${ }^{2}$ International Fellow, Walsall Manor Hospital, West Midlands, UK

${ }^{3}$ Assistant Professor, Liaqat National Hospital, Karachi, Pakistan

${ }^{4}$ Work Experience Student, Walsall Manor Hospital, West Midlands, UK

*Corresponding Author: Munawar Shah, Consultant Orthopaedic Surgeon, Walsall

Manor Hospital, Senior Author, Supervision, West Midlands, UK.
Received: November 30, 2020

Published: January 16, 2021

(C) All rights are reserved by Munawar

Shah., et al.

\begin{abstract}
Background: The aim of study is to evaluate the effectiveness of surface marking in determining working portal for acromioclavicular joint pathology during shoulder arthroscopy.

Method: This prospective study was conducted in Walsall Manor Hospital from January 2015 to December 2017 during which 47 patient of shoulder arthroscopy was admitted with diagnosis of acromioclavicular joint pathology. Surface marking of acromioclavicular joint was done in every patient with the technique described below before starting shoulder arthroscopy, which later used for making ACJ portal.

Result: Out of 47 patient we were able to reach acromioclavicular joint in 45 patients with help of surface marking before shoulder arthroscopy which results in decrease in intraoperative time and complications in shoulder arthroscopy.

Conclusion: Surface marking of acromioclavicular joint by described technique is helpful in making acromioclavicular joint decompression portal during shoulder arthroscopy.
\end{abstract}

Keywords: Acromioclavicular Joint (ACJ); Surface Marking; Shoulder Arthroscopy

\section{Introduction}

Subacromial impingement is one of the most common cause of shoulder pain. It limits daily living activities and usually non responsive to conservative treatment. The range of pathology is from sub acromial bursitis to tendinopathy and rotator cuff tears [1]. Sub acromion impingement can be diagnosed easily by clinical examination (painful movement of arch and weakness of external rotation) [2] or by radiological method like ultrasound or MRI [3] and supplemented by subacromial diagnostic block [4]. Arthroscopic
Subacromial decompression is the treatment of choice as to remove physical cause of impingement which is usually a curved acromion or ACJ arthritis. The most important step in decompression is the identification of anatomy of subacromial space. Acromioclavicular joint is one of the most important landmarks which should be identified and debrided. There are various ways of identification but in this article, we have described very convenient surface anatomical landmarks which will help identify the ACJ without much difficulty arthroscopically. 
There are various ways of dealing with ACJ pathology, either to approach the joint while doing subacromial decompression or direct approach via anterosuperior or posterosuperior portals. Exact localisation of the ACJ without hassle remains the first essential step to approach ACJ. It is sometimes difficult to localise the joint even after multiple needle insertions.

\section{Method and Study}

This study was conducted in WALSALL MANOR HOSPITAL, WALSALL UK. The duration of study was two years from January 2016 to December 2017. During this period patients which were presented in clinic with shoulder pain were evaluated and patients who had acromioclavicular joint pathology (mainly impingement) were included in the study. Total 93 patients were selected from the clinic which were conservatively managed for 6 months by physiotherapy and oral analgesia. Those who responded to conservative management were excluded from the study, 57 patients did not get relief from the conservative management and were offered shoulder arthroscopy and subacromial decompression but only 47 patients gave consent for procedure.

Inclusion criteria for study were; patient having shoulder pain with impingement test positive (painful arc with weakness of external rotation), patients got relieved with subacromial blockade and presence of acromioclavicular joint pathology on radiology (ultrasound or MRI).

Exclusion criteria were history of previous shoulder surgery of effected shoulder, history of instability, sign of infection on skin and shoulder.

In 47 patients shoulder arthroscopy was performed by experience arthroscopic surgeon in beech chair position by using standard portal, but before starting shoulder arthroscopy we have developed a surface marking technique to exactly localise the ACJ. The inner border of acromion, an extension of the spine of scapula and clavicle is marked. The point where they meet a perpendicular line is drawn. Coracoid is marked separately and a horizontal line is drawn until it meets with the vertical line at 90 degrees. At the intersection under the inferior margin of clavicle is portal for ACJ.

A needle is inserted from the skin in order to localize the ACJ with arthroscope. Standard posterior arthroscopy portal is made and the needle is visualised in the rotator interval. The needle is withdrawn and a size 11 blade is used to complete the portal. This portal can be used for instrumentation of subacromial decompression and ACJ resection.

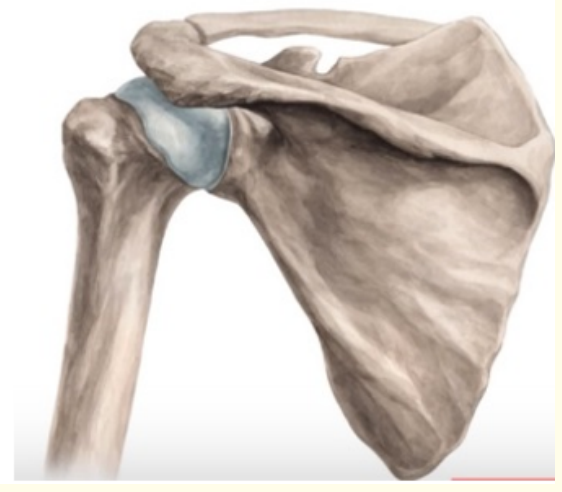

Figure 1: Osseous Anatomy of the Shoulder.

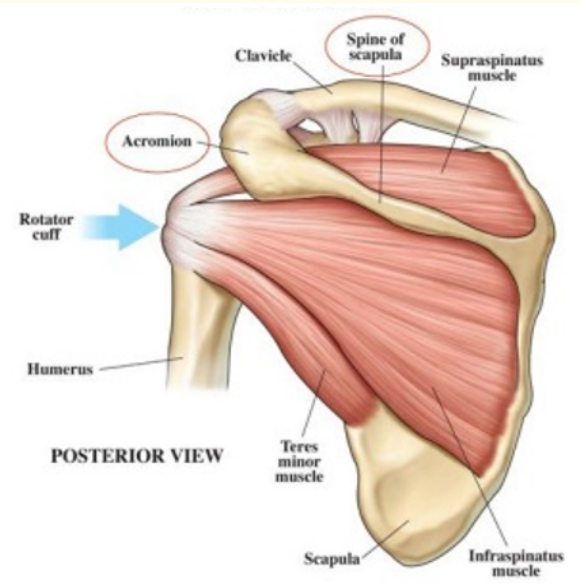

Figure 2: Muscular Anatomy of Shoulder.
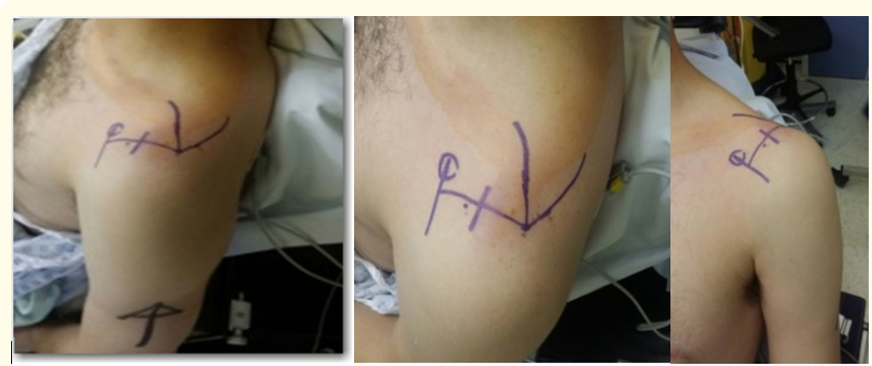

Figure 3: (A) Inner Border of Acromion and clavicle is marked. Vertical line is drawn. (B) Coracoid is marked and horizontal line is drawn intersecting the vertical line. (C) Under the Clavicle is portal for ACJ. 


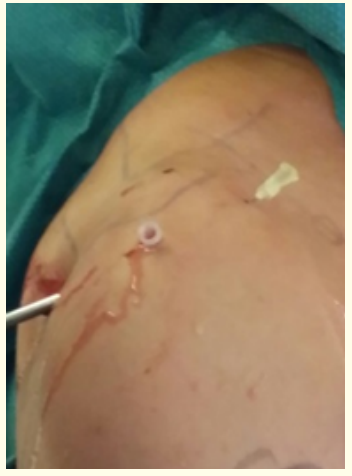

Figure 4: Needle inserted at ACJ.
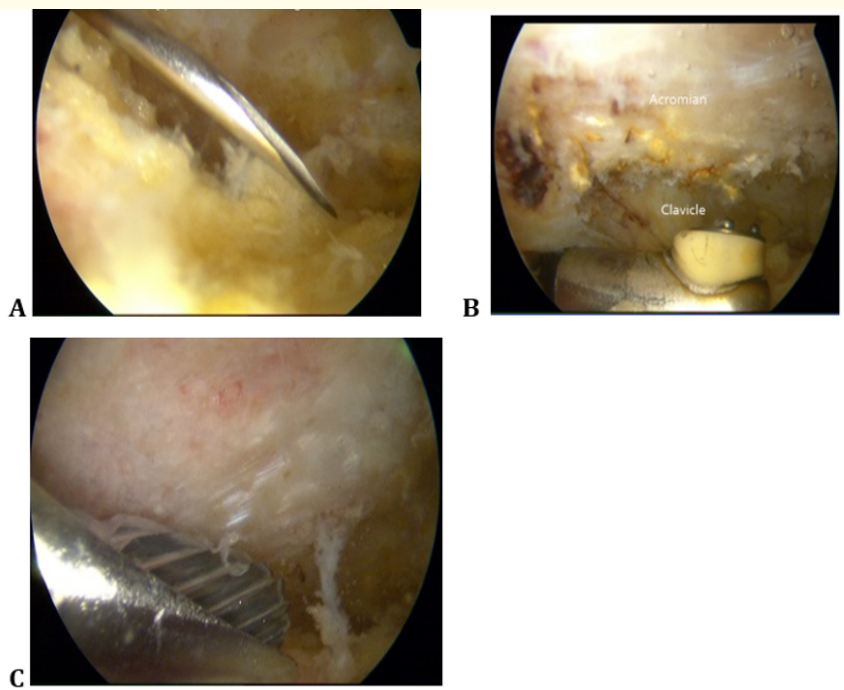

Figure 5: (A) Arthroscopic view of Needle. (B) Cleaning of under surface (C) Decompression using burr.

\section{Result}

We had done shoulder arthroscopy in 47 patients of acromioclavicular joint pathology in all of which surface marking of acromioclavicular joint was by above described technique before starting shoulder arthroscopy and we were managed to reach acromioclavicular joint in 45 patients and caused decompression of ACJ by using that portal, which proved that surface marking by above described method is a useful technique in creating portal for acromioclavicular joint pathology.

\begin{tabular}{|c|c|}
\hline \multicolumn{2}{|c|}{04} \\
\hline Total no of patients for arthroscopy & $\mathbf{4 7}$ \\
\hline Mean age & $42(+/-6.8)$ \\
\hline Male/female & $33 / 14$ \\
\hline Body mass index & $27.3(+/-2.4)$ \\
\hline Mean shoulder arthroscopy time & 47 MINS (+/- 5.7) \\
\hline
\end{tabular}

Table 1

\section{Discussion}

The acromioclavicular joint pathology is a significant source of shoulder pain. It can be either a degenerative disease [5] of the ACJ on its own causing impingement and cuff deterioration or a hyperaemic bone resorption giving rise to shoulder symptoms. However, most of the times it is linked to the subacromial pathology. Due to diffuse nature of pain in the shoulder related to various structures it is at time difficult to clearly identify the exact pathology [6]. It is at time necessary to inject AC joint as a diagnostic modality. The landmark is useful to confirm diagnosis as part of neers test. Some methods are described in the literature [7] to identify the landmark arthroscopically. But it is still difficult to identify the AC joint with accuracy for which we devised this method.

Shoulder arthroscopy is a common procedure performed for acromioclavicular joint pathology like sub acromial decompression [8] but in no previous study [9], surface marking of acromioclavicular joint was done before starting shoulder arthroscopy and most of study had used fixed point ( in line with anterior acromion and 2 to $3 \mathrm{~cm}$ distal to lateral edge of acromion ) for creating portal for decompression of acj, with help of insertion of needle during shoulder arthroscopy [10] which sometime becomes difficult and cause troublesome problem for operative surgeon and increased operative time. Also placing portal too high or low can cause problem in decompression or damaged to neurovascular structure (axillary nerve) [11].

\section{Conclusion}

Subacromial decompression is the treatment of choice for impingement syndrome. The essential component of decompression is the identification of subacromial anatomy which includes identification of acromioclavicular joint along with other structures. At times it gets very difficult to identify the acromioclavicular joint arthroscopically. The anatomical landmark described is very reliable 
and provides accurate identification of the acromioclavicular joint for subacromial decompression.

\section{Bibliography}

1. Tauber M. "Diseases of the acromioclavicular joint". Der Orthopade 45.6 (2016): 555-568.

2. De Witte PB., et al. "Study protocol subacromial impingement syndrome: the identification of pathophysiologic mechanisms (sistim)". BMC Musculoskeletal Disorders 12.1 (2011): 282.

3. Javed S., et al. "A simple method for identifying the acromioclavicular joint during arthroscopic procedures". Journal of Hand and Microsurgery 6.1 (2014): 26.

4. Huisstede BM., et al. "Multidisciplinary consensus on the terminology and classification of complaints of the arm, neck and/or shoulder". Occupational and Environmental Medicine 64 (2007): 313-319.

5. Park HB., et al. "Diagnostic accuracy of clinical tests for the different degrees of subacromial impingement syndrome". Journal of Bone and Joint Surgery 87 (2005): 1446-1455.

6. Silva L., et al. "Accuracy of physical examination in subacromial impingement syndrome". Rheumatology 47 (2008): 679-683.

7. Neer CS. "Impingement lesions". Clinical Orthopaedics and Related Research 173 (1983): 70-77.

8. Micheal P., et al. "Arthroscopic shoulder decompression". Operative Techniques in Orthopaedics 25 (2015): 10-14.

9. Kevin W Farmer., et al. "Shoulder arthroscopy: the basic". Journal of Bone and Joint Surgery 40.4 (2015): 817-821.

10. E Scott Paxton., et al. "Shoulder arthroscopy basic principle of positioning anaesthesia and portal anatomy". Journal of the American Academy of Orthopaedic Surgery 21 (2013): 332342.

11. Lars Aage., et al. "Arthroscopic shoulder decompression results after two year". Danish Medical Journal 62.3 (2015): A5026.

\section{Assets from publication with us}

- Prompt Acknowledgement after receiving the article

- Thorough Double blinded peer review

- Rapid Publication

- Issue of Publication Certificate

- High visibility of your Published work

Website: https://www.actascientific.com/

Submit Article: https://www.actascientific.com/submission.php Email us: editor@actascientific.com

Contact us: +919182824667 